\title{
Antiproton and Electron Optics for Electron Cooling
}

\author{
A. Burov, \\ Fermi National Accelerator Laboratory \\ P.O. Box 500, Batavia, Illinois 60510
}

June 26, 2000

\begin{abstract}
Optical issues for electron cooling in the Recycler [1,2] are discussed. An optimal value of the antiproton beta-function depends on the cooling purposes (longitudinal or transverse), on the antiproton emitances and the energy spread. A proposal is suggested for the optimal optics at the so-called SS30HB interval in the Recycler, where electron cooling is supposed to be applied. Both electron cooling and the phase trombone requirements are taken into account. The electron optic scheme is also presented.
\end{abstract}

\section{How High Is the Optimal Beta?}

Choice of a proper optics for electron cooling depends on the beam specifics and on the possibilities and purposes of the cooling process. It depends on

- whether a longitudinal or transverse cooling or both of them are going to be applied;

- the cooled beam emittances,

- the alignment and electron current limitations.

*e-mail: burov@fnal.gov, tel: (630)840-8852,fax: (630)840-6311 
The optimal choice is sensitive to the specifics of the cooling scenario and changes with it.

Assuming the electron angles to be small enough, the transverse cooling rate writes

$$
\lambda_{\perp} \propto \frac{I_{e} l_{c}}{r_{\perp}^{2}\left(2 v_{\perp}^{2}+v_{\|}^{2}\right)^{3 / 2}} \propto \frac{\beta_{c}^{1 / 2}}{\varepsilon_{n}^{3 / 2}\left(2+v_{\|}^{2} / v_{\perp}^{2}\right)^{3 / 2}}
$$

where $v_{\|}$is the pbar longitudinal velocity, $v_{\perp}=\sqrt{\varepsilon_{n} \gamma \beta / \beta_{c}}$ is the transverse velocity; both are taken in the beam frame. The rate is maximal over the beta-function $\beta_{c}$ when

$$
v_{\perp}=v_{\|} \cdot
$$

Substituting the rms parameters, it gives the optimal beta-function

$$
\beta_{c}=\frac{\varepsilon_{n} \gamma}{(\Delta p / p)^{2} \beta}
$$

expressed in terms of the rms momentum spread $\Delta p / p$ and normalized emittance $\varepsilon_{n}$.

When the Recycler was constructed, there was no detailed electron cooling design for the Fermilab, and the transverse cooling was considered as a main goal. Following the SSC MEB electron cooling design, the beta-function at the cooling section was picked at $\beta_{c}=200 \mathrm{~m}$.

Assuming the transverse cooling in the Recycler deals with the pbar beam occupying the longitudinal phase space $\mathcal{A}=4(\Delta p / p) E_{0} T_{0}=150 \mathrm{eVs}$, with the normalized rms emittance $\varepsilon_{n}=5 \pi \mathrm{mm}$ mrad, it gives for the transverse optimum $\beta_{c}=300 \mathrm{~m}$, close to the picked number above. However, this number depends quadratically on the longitudinal emittance. An updated scenario is based on the longitudinal phase space $\mathcal{A}=400 \mathrm{eVs}$ [2], and this results in the transverse optimal beta-fenction $\beta_{c}=40 \mathrm{~m}$. A transverse stochastic precooling would additionally reduce this value.

The longitudinal rate

$$
\lambda_{\|} \propto \frac{I_{e} l_{c}}{r_{\perp}^{2}\left(v_{\perp}^{2}+v_{\|}^{2}\right) v_{\|}} \propto \frac{1}{\varepsilon v_{\|}\left(\pi+v_{\|}^{2} / v_{\perp}^{2}\right)}
$$

is independent on the beta-function when $v_{\perp} \gg v_{\|}$. Otherwise, the rate goes down when the beta-function increases.

Dependence of the cooling rates on the beta-function is presented in Fig.1.

The disadvantages of the high beta-function are 


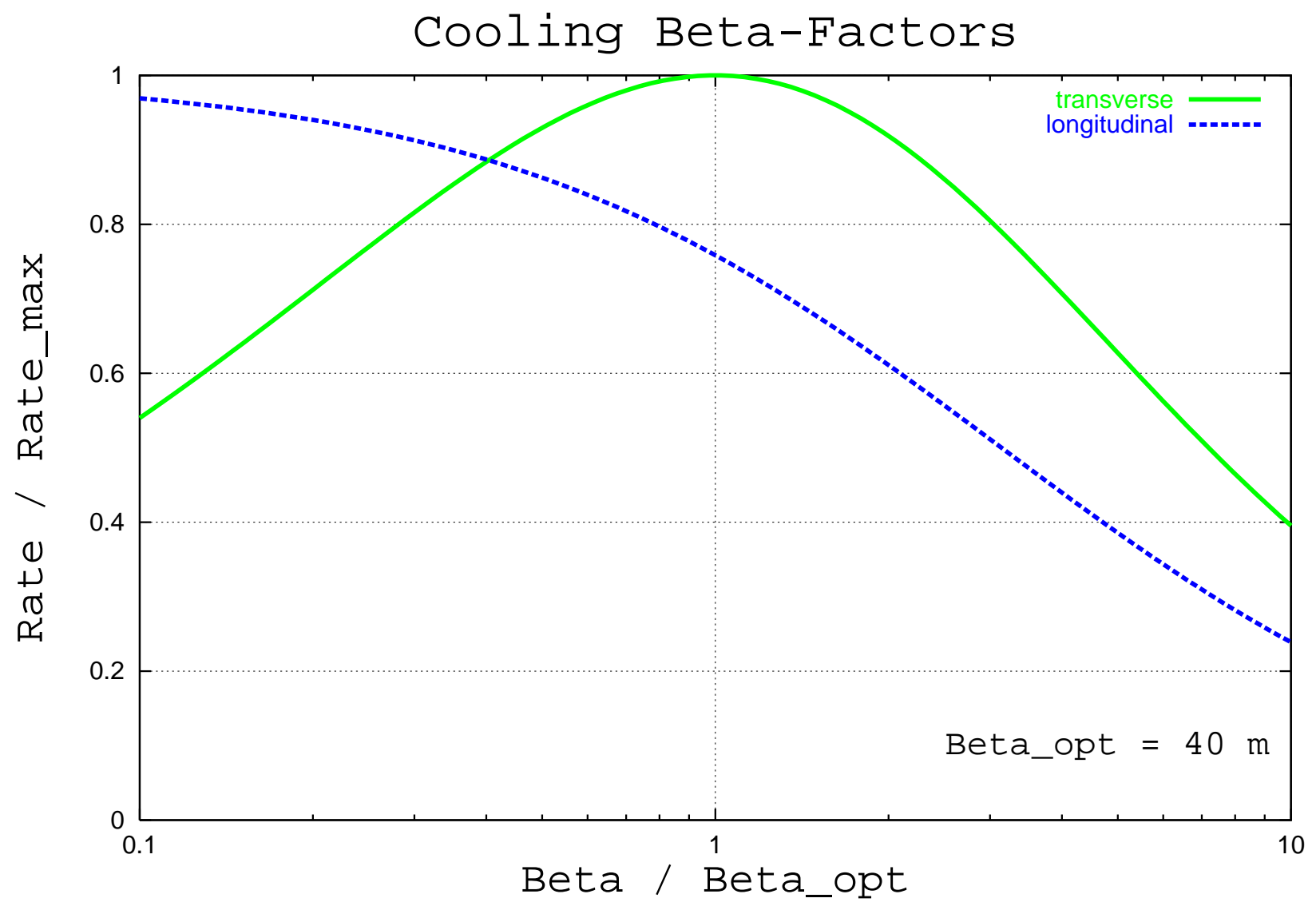

Figure 1: Dependence of the cooling rates on the beta-function 
- More difficult requirements on the quality of the electron beam and $e-\bar{p}$ alignment.

- Reduced dynamic aperture of the antiprotons in the Recycler.

Thus, for purposes of the longitudinal cooling, the beta-function should be taken as low as possible, what is the length of the cooling section, $\beta_{c}=l_{c}$. The transverse cooling weakly depends on the beta-function: a two-times reduction of the beta-function from the optimal value decreases the rate by $7 \%$ only.

Taking into account all these factors, namely

- High longitudinal phase space ( 100 bunches scenario)

- Expected transverse stochastic cooling / precooling

- Weak dependence of the transverse cooling on the beta-function

- Longitudinal rate is $\approx 25 \%$ reduced at the transverse optimum

- Not the least: tough alignment and dynamic aperture issues

the value of the beta-function of $\beta_{c}=24 \mathrm{~m}$ was picked, for the cooling length $l_{c}=20 \mathrm{~m}$

Thus, a re-design of the optics in the cooling section is needed. It has to satisfy the following conditions:

1. The cooling interval of $20 \mathrm{~m}$ is located at a given part of the $140 \mathrm{~m}$ insertion. In the middle of it, $\beta_{x, y}=\beta_{c}=24 \mathrm{~m}, \alpha=0$.

2. At the boundaries of the insert, $\beta_{x, y}, \alpha_{x, y}$ do not change.

3. Within the insert, $\max \beta_{x, y} \leq 80 \mathrm{~m}$.

4. The same or reflected working point $\left\{\nu_{x}\right\},\left\{\nu_{y}\right\}$ is desirable to be achieved.

5. The space of the insert is used for a phase trombone. The tune width of the trombone has to be high.

The solution has been found by means of the OptiM program [3] ; this code is designed as a $M S$ Windows application; it is used as a main code for optical calculations at Jefferson laboratory.

The current and the proposed optical schemes for the antiprotons at the socalled SS30HB section of the Recycler are presented in Figs. 2 and 3 respectively. The possibilities of the phase trombone are shown in Fig. 4 with the current working point marked by an arrow. 


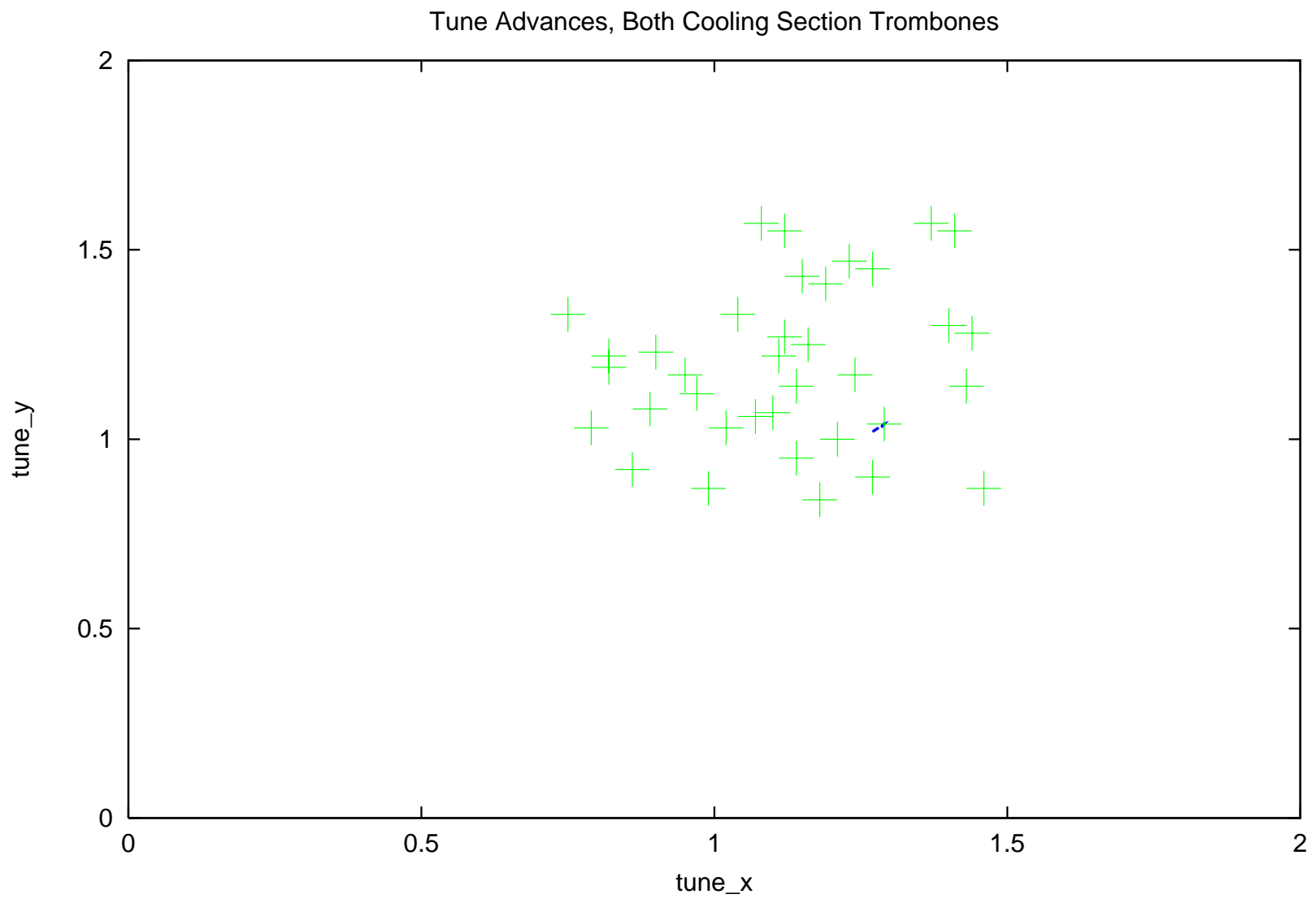

Figure 4: The possibilities of the phase trombone. The current working point is marked by an arrow. 


\section{Electron Beam Requirements}

To be an effective cooler, an electron beam has to satisfy certain requirements.

1. The current must be high enough; for $20 \mathrm{~m}$ of the cooling interval $I_{e} \geq$ $300 \mathrm{~mA}$.

2. Electron beam has to have an optimal radius, $a_{e}=6.5 \mathrm{~mm}$ for the updated choise of the $\beta$-function.

3. Electron angles in the cooling interval have to be not higher than $70 \mu \mathrm{rad}$.

A solenoid magnetic field in the cooling section allows to have both focusing and small angles. To avoid an excitation of the Larmor rotation in the cooler, the magnetic flux through the beam at the cathode must be the same as in the cooler: $\left.B a_{e}^{2}\right|_{\text {cathod }}=\left.B a_{e}^{2}\right|_{\text {cooler }}=\Phi / \pi$.

For $600 \mathrm{G}$ at the cathode with the radius of $2.5 \mathrm{~mm}$, it gives $100 \mathrm{G}$ in the cooling interval.

This scheme also requires a rotation-invariance of the beam phase-space transformation at the transport from the gun to the cooler. This is naturally satisfied with solenoid lenses as focusing elements and bending magnets with the field in$\operatorname{dex} n=1 / 2$. The invariance of the electron transport can be also provided in a different way. Namely, bending blocks can be constructed from the conventional ( $n=0$ ) dipoles and quadrupoles, so that the mapping of the whole block is rotationally invariant. With the bending magnets splitted with a proper focusing in between, these block can provide zero dispersion in the downstream line.

It is important that a continuous solenoid field is not required along the transport line [4]. It is sufficient to have this field $B(s)$ only locally, in the lenses. At the straight intervals, the beam envelope goes

$$
r^{\prime \prime}+\left(\frac{B(s)}{2 B \rho}\right)^{2} r-\epsilon_{e}^{2} / r^{3}=0
$$

as if $\epsilon_{e}=\Phi /(2 \pi B \rho)$ is its emittance; the value $\beta_{e}=r^{2} / \epsilon_{e}$ can be referred to as an effective beta-function of the electron beam.

An envelope of the electron beam is presented in Fig. 5. All the bending blocks are invariant and provide zero dispersion downstream.

When lens-to-lens intervals $L$ are not high, $L \leq \beta_{e}$, the beta-function is almost constant,

$$
\beta_{e}=\left(\left\langle\left(\frac{B(s)}{2 B \rho}\right)^{2}\right\rangle\right)^{-1 / 2}
$$


so the smooth approximation can be used.

Electron angles $\theta_{e}$ start to influence the cooling process if $\theta_{e} \geq 70 \mu \mathrm{rad}$. Possible perturbations of the electron trajectories are listed below

- Stray magnetic fields, misalignments, errors of solenoid lenses and bending magnets. The transport line has to be shielded, the optical elements tested and aligned, the correctors to be tuned.

- The lens aberrations are described by a single parameter, the aberration coefficient $a_{*}$ such that the focal strength of the lens $f(r)$ as a function of the offset goes as $f(r)=f(0)\left(1+r^{2} / a_{*}^{2}\right)$. For a solenoid lens with the length $l \ll 1 / f$ and radius $b \ll l$ the aberration coefficient $a_{*}=1.8 \sqrt{b l}$. A perturbation of the focal strength $\delta f$ leads to the angle oscillations near the unperturbed trajectory with the amplitude

$$
\delta \theta=\frac{\epsilon_{e}}{r} \frac{\delta f}{2 f} .
$$

Assuming the lens' radius $b=2 \mathrm{~cm}$, it gives $\delta \theta=2 \cdot 10^{-6}$ which is negligible in this scale.

- The aberrations in the bending magnet are of the sextuple type; they are described by 32 matrix elements like $\left(x^{\prime} \mid x y^{\prime}\right),(y \mid x y)$ etc. The aberrations are minimized when the beam beta-function in the magnet is equal to the bending radius, which requires $3 \mathrm{~mm}$ of the beam radius. In this case, the angle is $3 \cdot 10^{-5}$.

- Space charge of the electrons, trapped ions and antiprotons causes the drift in the cooling section. The drift of the electrons due to their own space charge gives the angle $\theta_{e e}=2 \pi n_{e} e a_{e} /\left(\gamma^{2} B\right)$.

For $I_{e}=0.5 \mathrm{~A}, B=50 \mathrm{G}$, it gives $\theta_{e e}=3 \cdot 10^{-5}$.

The drift on the trapped ions is enhanced as $\gamma^{2} n_{i} / n_{e}$. So their relative concentration $n_{i} / n_{e}$ has to be not more than $2 \%$.

For the number of antiprotons in the Recycler $N_{\bar{p}}=10^{13}$ with the beam radius $a_{\bar{p}}=1 \mathrm{~mm}$, the electron drift on their space charge would give the same number, $\theta_{e \bar{p}}=3 \cdot 10^{-5}$.

- Cathode temperature $T_{c}=0.2 \mathrm{eV}$ contributes the rms angle $\theta_{T}=2.5 \cdot 10^{-5}$. 


\section{Summary}

- The required cooling length, electron current and beta-function are specified.

- A solution for antiproton optics in the MI-30 region of the Recycler is suggested. It incorporates the electron cooling and wide width phase trombone.

- The electron transport line is designed. The requirements are formulated, and the various sources of aberrations are estimated.

\section{References}

[1] S. Nagaitsev, A. Burov, A. C. Crawford, T. Kroc, J. MacLachlan, G. Saewert, C. W. Schmidt, A. Shemyakin and A. Warner, "FNAL R\&D in medium energy electron cooling", NIM-A, 441, p. 241 (2000).

[2] A. Burov, J. MacLachlan, J. Marriner and S. Nagaitsev, "Scenario for electron cooling of antiprotons in the Recycler", ibid. p. 234.

[3] A homepage of OptiM is at http://host.sybercom.net/users/ldbs .

[4] A. Burov, Ya. Derbenev, S. Nagaitsev and A. Shemyakin "Optical Principles of Beam Transport for Relativistic Electron Cooling", FERMILAB-PUB-00100-T (2000), submitted to PRST-AB. 


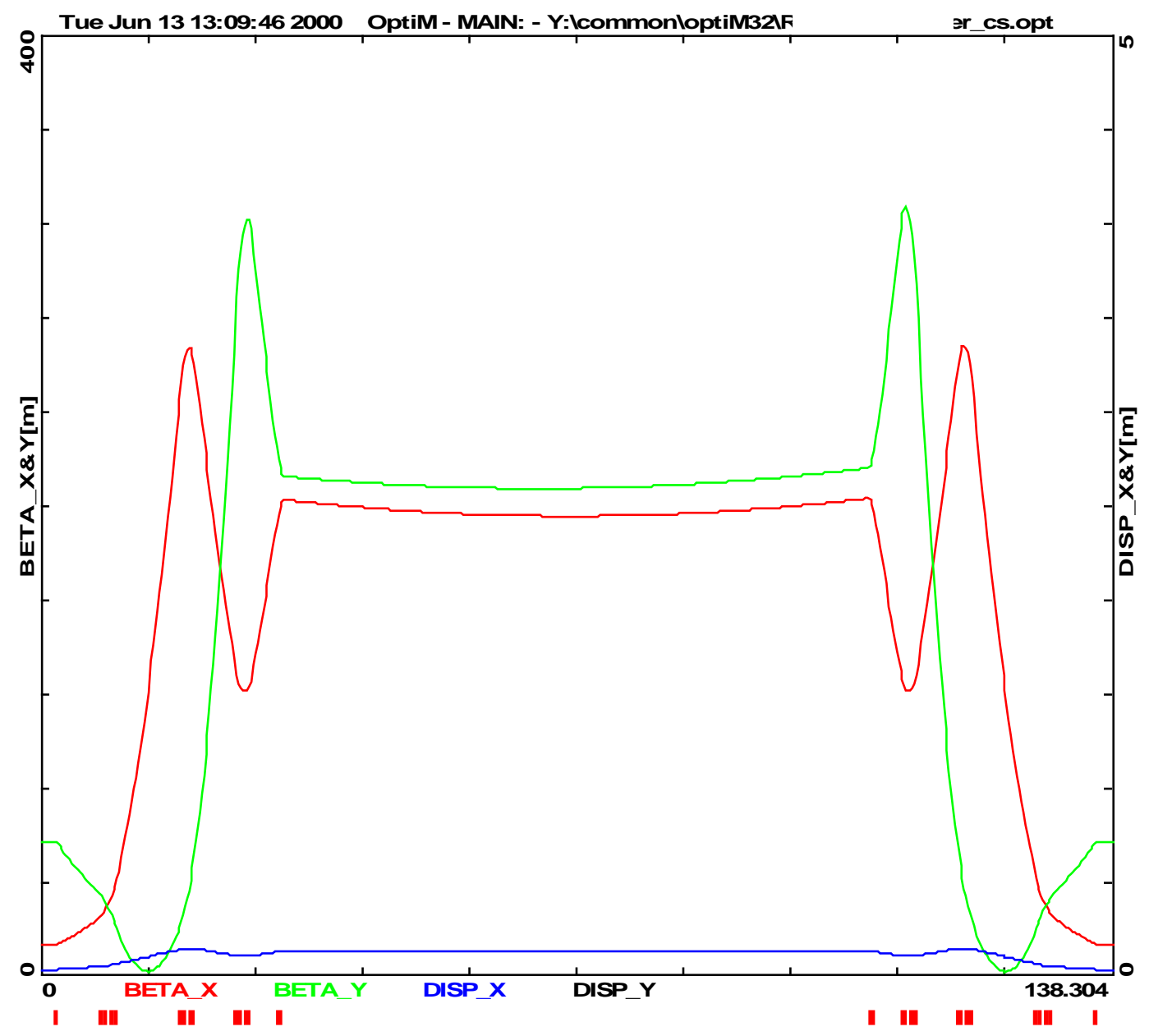

Fig. 2. Recycler SS30HB section, the current state. The phase advances: $Q_{x}=0.29, \quad Q_{y}=1.04$ 


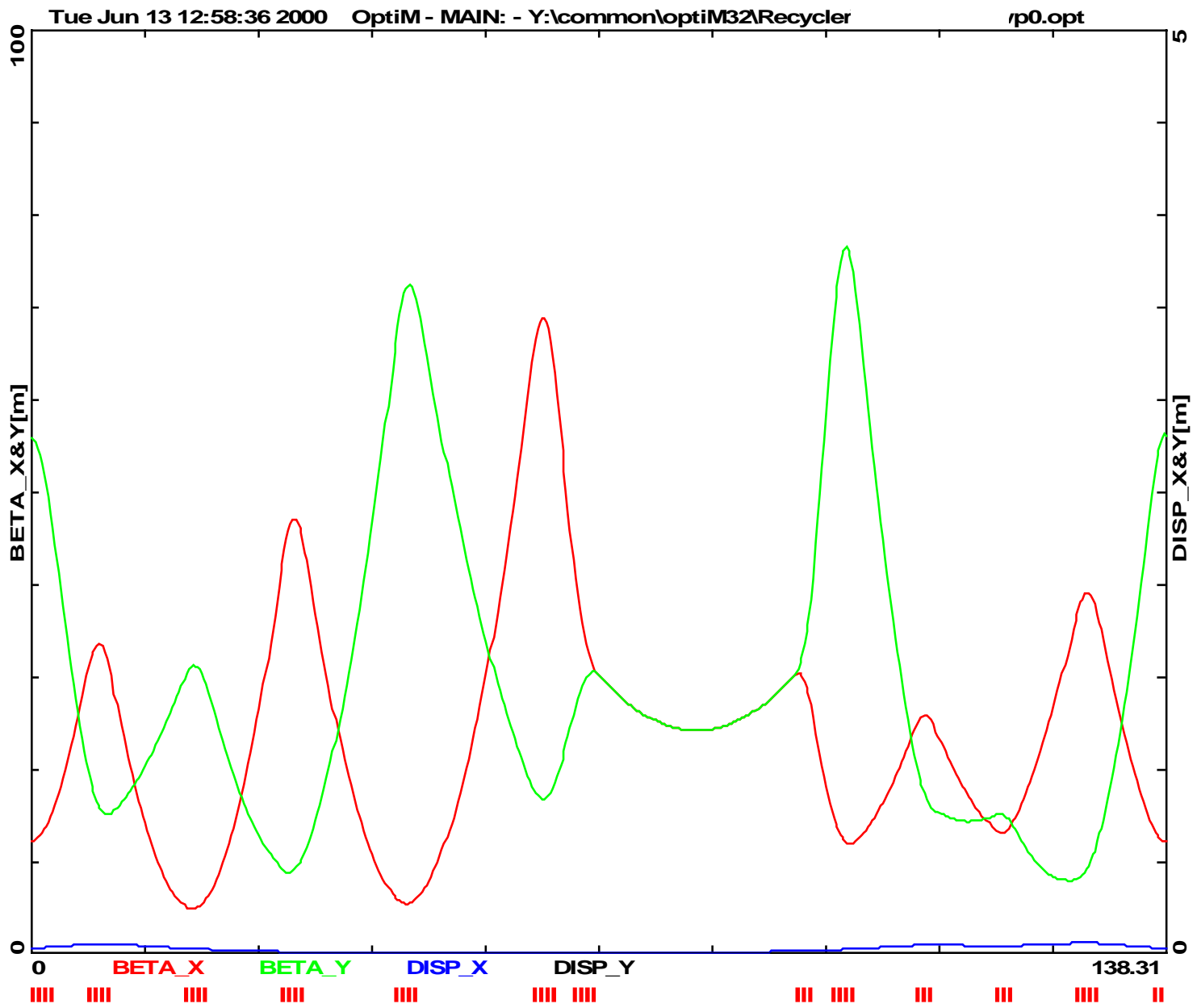

Fig. 3. Recycler SS3OHB section, as proposed. Electron cooling interval of $20 \mathrm{~m}$ and the phase trombone are assumed. The quads are tuned to the same phase advance: $Q_{x}=1.29, \quad Q_{y}=1.04$ 


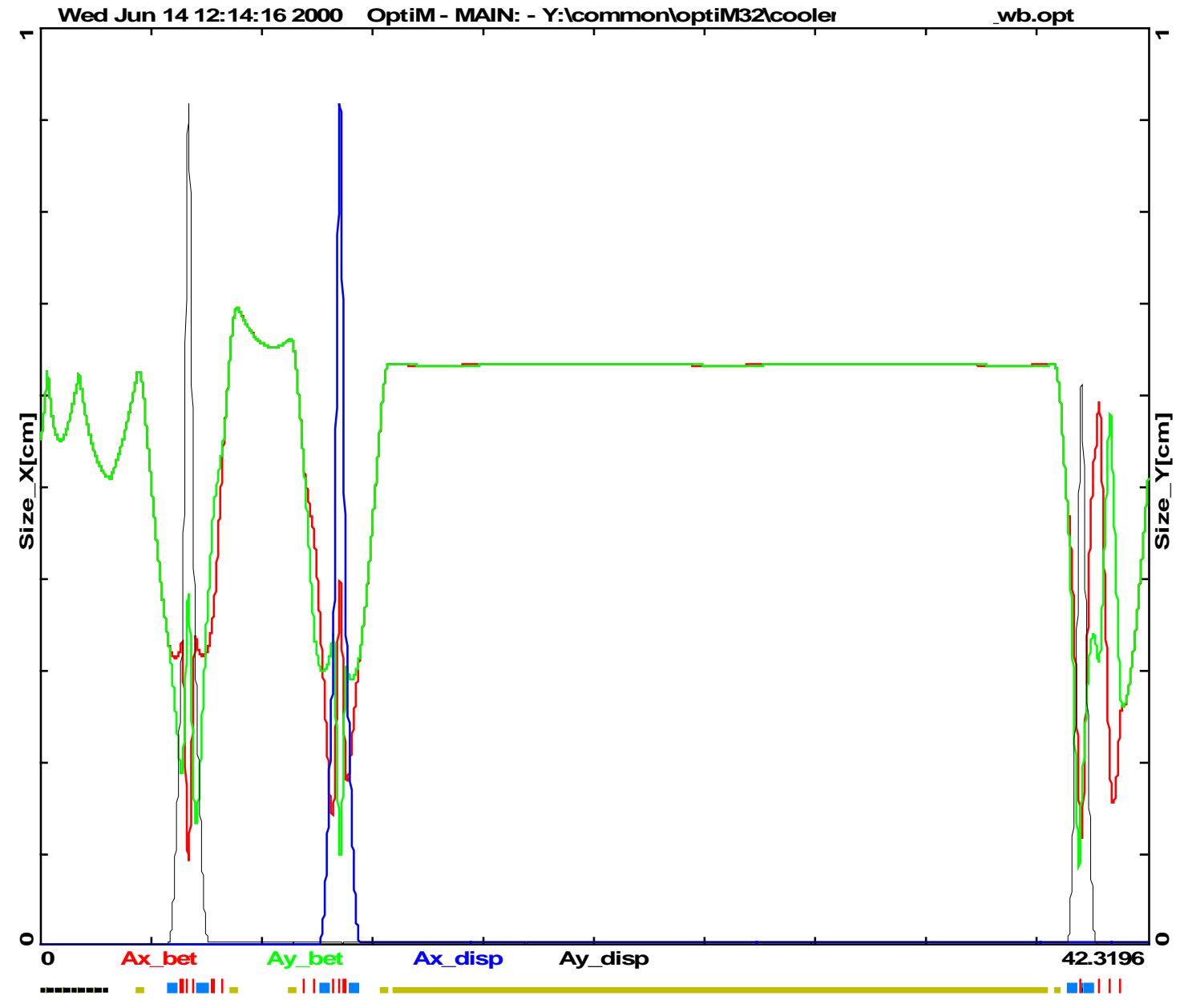

Fig. 5. Electron beam envelope and dispersion at the transport from the Pelletron to the beginning of the return line. Below, the black color corresponds to the electrostatic acceleration, yellow - to the solenoids, blue - to the dipoles, and red - to the quads. All the bending blocks are seen as rotation-invariant and achromatic. Maximal dispersion is $63 \mathrm{~cm}$. 